Mens

revue d'histoire intellectuelle de l'Amérique française

MENS

\title{
Un réseau indépendantiste dans le Québec des années 1930
}

\section{Mathieu Noël}

Volume 11, numéro 2, printemps 2011

URI : https://id.erudit.org/iderudit/1023373ar

DOI : https://doi.org/10.7202/1023373ar

Aller au sommaire du numéro

Éditeur(s)

Centre de recherche en civilisation canadienne-française

ISSN

1492-8647 (imprimé)

1927-9299 (numérique)

Découvrir la revue

Citer cet article

Noël, M. (2011). Un réseau indépendantiste dans le Québec des années 1930. Mens, 11(2), 69-97. https://doi.org/10.7202/1023373ar

\section{Résumé de l'article}

Dans les années 1930, l'idée d'indépendance du Québec est proposée et défendue par des groupes de jeunes nationalistes tels que les Jeune-Canada, les Jeunesses patriotes et les collaborateurs des périodiques Vivre et La Nation. Ces différents groupes s'inscrivent dans un réseau. Les indépendantistes entretiennent des liens entre eux et souhaitent transmettre leurs idées à la société canadienne-française, mais ils ne réussissent pas à rejoindre les masses et ainsi transformer leur réseau en mouvement social. Cet article présente et analyse le réseau indépendantiste des années 1930 afin de comprendre son développement, les raisons qui l'ont empêché de devenir un mouvement social et les causes de sa disparition vers la fin de la décennie. 


\title{
Un réseau indépendantiste dans le Québec des années 1930
}

\author{
Mathieu Noël \\ Université du Québec à Montréal
}

\section{Résumé}

Dans les années 1930, l'idée d'indépendance du Québec est proposée et défendue par des groupes de jeunes nationalistes tels que les JeuneCanada, les Jeunesses patriotes et les collaborateurs des périodiques Vivre et La Nation. Ces différents groupes s'inscrivent dans un réseau. Les indépendantistes entretiennent des liens entre eux et souhaitent transmettre leurs idées à la société canadienne-française, mais ils ne réussissent pas à rejoindre les masses et ainsi transformer leur réseau en mouvement social. Cet article présente et analyse le réseau indépendantiste des années 1930 afin de comprendre son développement, les raisons qui l'ont empêché de devenir un mouvement social et les causes de sa disparition vers la fin de la décennie.

\section{Abstract}

During the 1930s, the idea of Quebec independence was promoted and defended by several groups of young nationalists, including the JeuneCanada, the Jeunesses patriotes, and contributors to the journals Vivre and La Nation. These different groups formed something of a network. They maintained ties to each other and sought to promote their ideas within French Canadian society. However, they were unable to achieve any real popular support and thus failed to transform their network into a wider social movement. This article presents and analyses the independentist network of the 1930s in order to better understand its development, along with the reasons that prevented it from becoming a 
social movement and the factors that brought about its disappearance in the latter years of the decade.

Il est de plus en plus connu que l'idée d'indépendance du Québec ne débute pas au tournant des années 1960 avec l'apparition d'organisations telles que l'Alliance laurentienne et le Rassemblement pour l'indépendance nationale. En effet, l'indépendantisme est déjà discuté et débattu dans les cercles nationalistes des années $1930^{1}$. L'idée est portée principalement par des groupes de jeunes qui se réclament du groulxisme et qui y voient une solution aux désordres causés par la crise économique. Quelques historiens se sont intéressés à ces organisations. Pensons aux travaux d'André-J. Bélanger, de Denis Chouinard et de Lucienne Fortin sur Jeune-Canada ${ }^{2}$, aux recherches de Robert Comeau, de Michèle Larose, de Julien Fabvre et de CharlesPhilippe Courtois sur le journal La Nation ${ }^{3}$, puis, plus récemment, aux articles d'Yvan Lamonde et au premier tome du collectif Histoire

\footnotetext{
${ }^{1}$ À l'époque, le terme le plus souvent utilisé pour désigner l'idée d'indépendance est celui de "séparatisme ".

${ }^{2}$ André J.-Bélanger, L'apolitisme des idéologies québécoises : le grand tournant de 19341936, Sainte-Foy, Les Presses de l'Université Laval, 1974; Denis Chouinard, « Des contestataires pragmatiques : les Jeune-Canada, 1932-1938 ", Revue d'histoire de l'Amérique française, vol. 40, n 1 (été 1986), p. 5-28; Lucienne Fortin, "Les Jeune-Canada (1932-1936) ", dans Fernand Dumont et al., Idéologies au Canada français, 1930-1939, Québec, Les Presses de l'Université Laval, 1978, p. 215-235.

${ }^{3}$ Robert Comeau, Les indépendantistes québécois, 1936-1938, mémoire de maitrise en histoire, Université de Montréal, 1971, 2 vols.; Michèle Larose, Les Jeunesses patriotes et "La Nation", un courant politique d'extrême droite au Québec : 19341939, mémoire de maîtrise en histoire, Université du Québec à Montréal, 1984; Julien Fabvre, «La Nation: les groulxiens devant la tentation fasciste 1936-1939 ", Bulletin d'histoire politique, vol. $9, \mathrm{n}^{\circ} 2$ (printemps 2001), p. 40-52; Charles-Philippe Courtois, "Le séparatisme québécois des années 1930 et les non-conformistes", Bulletin d'histoire politique, vol. 16, $\mathrm{n}^{\circ} 2$ (hiver 2008), p. 287-302.

${ }^{4}$ Yvan Lamonde, «Les Jeunesses patriotes (1935-1939) et la position non séparatiste de l'abbé Groulx ", Bulletin d'histoire politique, vol. 17, n² 2 (hiver 2009), p. 179 189; Yvan Lamonde, «La rage de Vivre et les Cahiers noirs (1934-1935) ", Mens: revue d'histoire intellectuelle de l'Amérique française, vol. IX, $\mathrm{n}^{\circ} 2$ (printemps 2009), p. 187-206.
} 
intellectuelle de l'indépendantisme québécois ${ }^{5}$. Jusqu'ici, ces groupes ont généralement été étudiés séparément ${ }^{6}$. Une telle approche permet de mieux connaître la constitution et les idées de ces groupes, mais pas de vérifier la force et la portée qu'avait l'idée d'indépendance. Dans cet article, je propose d'aborder l'indépendantisme de ces années dans une perspective plus globale. Je crois que les différents groupes indépendantistes s'inscrivent dans un réseau. Celui-ci sera présenté, et son développement au cours des années 1930 sera étudié. L'approche basée sur l'analyse des réseaux aidera à expliquer, d'abord, pourquoi l'idée indépendantiste n'a pas réussi à rejoindre les masses et, ensuite, les causes de sa disparition vers la fin de la décennie. En d'autres mots, il s'agit d'éclairer les raisons qui ont empêché le réseau de se transformer en véritable mouvement social.

\section{Lindépendantisme dans le cycle d'action collective}

Les indépendantistes québécois des années 1930 tentent de se regrouper en organisations dans le but de mener une action collective. Selon le sociologue Charles Tilly, l'action collective est le résultat du processus de mobilisation des ressources en vue de la défense d'un intérêt collectif. L'action collective est composée de différents cycles de contestation qui incluent le groupe de pression, le réseau, le mouvement social et le groupe révolutionnaire. En partant de la conceptualisation du politologue Guillaume Courty, nous pouvons établir que les indépendantistes des années 1930 ne représentent pas un groupe de pression, un groupe d'influence ou encore un lobby, puisqu'ils ne souhaitent pas défendre des intérêts communs auprès des personnes et des institutions décisionnelles? ${ }^{7}$. Ils ont plutôt une

\footnotetext{
${ }^{5}$ Robert Comeau, Charles-Philippe Courtois et Denis Monière (dir.), Histoire intellectuelle de l'indépendantisme québécois, t. 1 : 1834-1968, Montréal, VLB éditeur, 2010.

${ }^{6}$ Yvan Lamonde a tenté une synthèse dans « La constellation nationaliste de 1930 à 1939 : côté lumière, côté ombre ", dans Yvan Lamonde et Denis Saint-Jacques (dir.), 1937 : un tournant culturel, Sainte-Foy, Les Presses de l'Université Laval, 2009, p. 3-13.

${ }^{7}$ Guillaume Courty, Les groupes d'intérêts, Paris, La Découverte, 2006.
} 
vision qu'ils souhaitent élargir à l'ensemble de la société canadiennefrançaise. D'ailleurs, plusieurs d'entre eux rejettent le système politique en place et ses représentants.

Par ailleurs, les indépendantistes ne forment pas un mouvement social au sens où le conçoit Charles Tilly. Celui-ci présente le mouvement comme une action instrumentale qui correspond à des fins politiques. Le mouvement vise à pénétrer le système politique afin de mettre en application ses idées et sa vision de la société. Pour déterminer la présence d'un mouvement social, il faut, selon Tilly, une synthèse conséquente de trois éléments : une campagne, un répertoire d'actions et le concept du "WUNC " $^{8}$. Celui-ci fait référence à la respectabilité des participants (Worthiness), 'à l'unité des participants (Unity), à l'importance en nombre des participants (Numbers) et à leur degré d'engagement (Commitment). Nous verrons dans cet article que les indépendantistes des années 1930 ne respectent pas l'ensemble de ces critères. De plus, certains aspects, telles que la conjoncture et la prise de mauvaises décisions, nuisent à l'émergence du mouvement.

Les indépendantistes se situent au " cycle " précédent de l'action collective, soit le réseau social. Celui-ci est une structure composée d'individus et/ou d'organisations reliés entre eux par des liens sociaux. En ce sens, l'approche basée sur l'analyse des réseaux me semble adéquate pour mieux connaitre les indépendantistes de cette période. Elle consiste à déterminer les acteurs du réseau (les nouds) et les liens qui les unissent afin de permettre une analyse globale. Cette méthode s'inspire des idées de littéraires comme Michel Lacroix?. Tout comme lui, je privilégie une approche qualitative et historique du réseau. L'analyse des réseaux sociaux est un " courant de recherche spécifique au sein des sciences humaines, pourvu d'un appareil notionnel et de

\footnotetext{
${ }^{8}$ Charles Tilly, Social Movements, 1768-2004, Boulder, Colorado, Paradigm Publishers, 2004, p. 53-54.

${ }^{9}$ Michel Lacroix, « Analyse des réseaux sociaux et interdisciplinarité dans les études québécoises ", Globe : revue internationale d'etudes québécoises, vol. 7, nº 1 (printemps 2004), p. 11-25.
} 


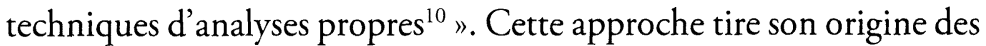
recherches d'Elizabeth Bott ${ }^{11}$ dans les années 1950, puis sa popularité a augmenté dans les années 1970 sous l'influence de la sociométrie et de l'anthropologie culturelle ${ }^{12}$. Les recherches de Stanley Milgram sur "l'expérience du petit monde " et surtout celles du groupe de recherche de Harrison White à l'Université Harvard, auxquelles participait notamment Mark Granovetter ${ }^{13}$, ont fait de la théorie des réseaux "l'un des secteurs les plus dynamiques de la sociologie contemporaine $^{14}$ ". Dans cet article, l'analyse des réseaux permet d'étudier les relations inter et intra-organisationnelles. Je propose de présenter et d'analyser le réseau indépendantiste afin de comprendre son développement, les raisons qui l'ont empêché de se transformer en mouvement social et les causes de sa disparition vers la fin de la décennie.

On peut diviser le réseau en deux périodes, 1930-1935 et 19351939, puisque l'année 1935 est une année charnière, tant dans la composition du réseau que dans les idées qui y sont dominantes. Néanmoins, il s'agit toujours d'un seul et même réseau, puisque les deux périodes partagent certains nœuds, mais surtout, plusieurs liens. De plus, même si la rhétorique, les moyens employés et la conception du nouvel État se transforment, c'est encore le même objectif qui est poursuivi, soit la création d'un État francophone indépendant en Amérique du Nord.

\footnotetext{
${ }^{10}$ Ibid., p. 12.

1 Elizabeth Bott, Family and Social Network: Roles, Norms and External Relationship in Ordinary Urban Families, London, Tavistock Publications, 1957.

${ }^{12}$ Lacroix, "Analyse des réseaux sociaux et interdisciplinarité dans les études québécoises ".

${ }^{13}$ Stanley Milgram, "The Small World Problem ", Psychology Today, vol. 1, n 1 (1967), p. 61-67; Harrison C. White, Chains of Opportunity: System Models of Mobility in Organizations, Cambridge, Harvard University Press, 1970; Mark Granovetter, "The Strength of Weak Ties: A Network Theory Revisited ", Sociological Theory, vol. 1 (1983), p. 201-233.

${ }^{14}$ Citation de Peter Hedström et Richard Swedberg traduite dans Lacroix, "Analyse des réseaux sociaux et interdisciplinarité dans les études québécoises ", p. 15.
} 


\section{0-1935 : le réseau sous l'influence jeune-canadienne}

Lidée de l'indépendance avait déjà été discutée lors des enquêtes annuelles de 1922 et de 1927 de la revue L'Action française ${ }^{15}$. Il n'est donc pas surprenant de retrouver au début des années 1930 des personnes favorables à cette idée. C'est le cas de Georges Boulanger qui, pendant l'hiver 1930, écrit des articles en faveur de l'indépendance dans la revue $\mathrm{La}$ Garde ${ }^{16}$. Toutefois, nous ne pouvons pas encore conclure à la présence d'un réseau indépendantiste, puisque ces individus sont isolés et n'agissent pas collectivement.

Il faut attendre la formation du groupe Jeune-Canada en décembre 1932, et plus précisément leur prise de position en faveur de l'indépendance à l'été 1933, pour qu'un réseau indépendantiste prenne réellement forme. Ce groupe est composé de quelques jeunes étudiants ${ }^{17}$ qui sont amis, pour la plupart, depuis leur rencontre au collège Sainte-Marie. En 1927, ceux-ci fondent le Cercle Crémazie afin d'organiser des tables rondes pour discuter de littérature et de politique. Ce cercle comprend André Laurendeau, Dollard Dansereau, Pierre Dansereau, Jean-Louis Dorais, Lucien L'Allier, Gilbert Manseau et Claude Robillard, tous de futurs membres de Jeune-Canada ${ }^{18}$. Puis, lors de leur passage à l'Université de Montréal, ils poursuivent cette tradition en fondant le Club X en $1931^{19}$. L'année suivante, sous les recommandations l'abbé Lionel Groulx, le Club X organise

${ }^{15}$ Ces enquêtes sont analysées par Pascale Ryan dans Penser la nation : la Ligue d'Action nationale 1917-1960, Montréal, Leméac éditeur, 2006.

${ }^{16}$ Georges Boulanger, "La puissance des groupements ", La Garde, vol. 1, n 3 (mars 1930), p. 2. La Garde est le mensuel de la Fédération catholique des gardes indépendantes du Canada, qui paraît de 1930 à 1932.

${ }^{17}$ En décembre 1932, la plupart des membres sont âgés de 20 à 25 ans. Précisons que dans les années de crise économique, la jeunesse canadienne-française émerge comme force politique et catégorie sociale plus autonome. À ce sujet, voir Louise Bienvenue, Quand la jeunesse entre en scène, Montréal, Éditions du Boréal, 2003.

${ }^{18}$ Lettre de François Rinfret à André Laurendeau, 12 juillet 1928, Bibliothèque et Archives nationales du Québec - Centre de Montréal (à l'avenir BAnQ-M), Fonds Familles Laurendeau et Perrault, CLG2/A5.

${ }^{19}$ Chouinard, «Des contestataires pragmatiques : les Jeune-Canada, 1932-1938». 
une première activité publique pour protester contre les injustices commises à l'endroit de la langue française. Dans le cadre d'une assemblée devant une salle comble au Gesù, à Montréal, le groupe lance le Manifeste de la jeune génération et une pétition pour les droits $\mathrm{du}$ français. Après ce premier succès, les jeunes choisissent de poursuivre leur militantisme public et adoptent le nom de Jeune-Canada. Trois présidents se succèdent à la direction du groupe : Pierre Dansereau (1932-1934), Jean-Louis Dorais (1934-1935) et Thuribe Belzile (1935-1936). Parmi les autres principaux membres, mentionnons Dominique Beaudin, George-Étienne Cartier, Pierre Dagenais, Dollard Dansereau, Paul Dumas, Gérard Filion, Bernard Hogue et André Laurendeau, auxquels se joignent par la suite certains de leurs amis tels qu'Émilien Brais, Robert Charbonneau, Lucien L'Allier, Dostaler O'Leary et Paul Simard.

À ce moment, le réseau est établi sur de solides bases, notamment en raison de la force des liens entre les différents acteurs et de son unité interne. La plupart des membres de Jeune-Canada sont amis depuis l'adolescence, tandis que d'autres se connaissent depuis l'enfance, puisque leurs parents étaient eux-mêmes des amis intimes. C'est le cas notamment des pères d'André Laurendeau, de Pierre Asselin et de Jean-Paul Verschelden, qui étaient tous les trois dans l'entourage de L'Action française. Parfois, les liens entre les membres de Jeune-Canada sont encore plus forts que l'amitié, il faut alors parler de liens familiaux. Le meilleur exemple est celui de GeorgeÉtienne Cartier ${ }^{20}$. Premièrement, il est le beau-frère de Jean-Louis Dorais, qui a épousé sa sœur Jacqueline. Pour sa part, George-Étienne s'est marié avec Georgette Simard, la sœur de Paul Simard. Une autre sœur de Paul, Paule Simard, a épousé Émilien Brais. Ajoutons à ce portrait Gérard Filion et Jean-Paul Verschelden, qui sont beaux-frères.

${ }^{20}$ Ces renseignements sur les liens de parenté mont été transmis par GeorgeÉtienne Cartier (1940-2010), le fils du membre de Jeune-Canada George-Étienne Cartier (1907-1983) et de Georgette Simard. À noter qu'il s'agit des descendants directs de George-Étienne Cartier (1814-1873), qui participa à la fondation de la Confédération en 1867. 
De plus, des liens supplémentaires se sont créés avec le temps, par exemple George-Étienne Cartier est devenu parrain du fils d'André Laurendeau, Olivier. Bref, nous pouvons retracer plusieurs unions entre les familles des membres de Jeune-Canada, ce qui renforce encore plus la cohésion entre ces amis du collège. Ces forts liens aident à comprendre pourquoi plusieurs membres resteront des amis intimes après la disparition de l'organisation.

Aussi, bien qu'elles ne soient pas admises dans le groupe, les femmes dans l'entourage de Jeune-Canada se réunissent pour militer et soutenir les actions de leurs conjoints ou de leurs frères. Mes recherches dans le Fonds Lionel Groulx m'ont permis de découvrir l'existence d'une aile féminine de Jeune-Canada : les JeuneLaurentienne - à ne pas confondre avec le groupe du même nom fondé en 1936 par Rosaire Morin et Paul-Émile Robert ${ }^{21}$. En novembre 1935, la présidente de Jeune-Laurentienne est Gabrielle Lévesque (belle-sœur de Dostaler O'Leary). Les autres membres sont Louise Mathys, Lucille Lévesque (amie de Dostaler O'Leary), Georgette Simard (søur de Paul Simard et épouse de George-Étienne Cartier), Lucille Berthiaume, Marguerite O'Leary (sœur de Dostaler O'Leary), Madeleine Dorais, Jacqueline Dorais (sœur de GeorgeÉtienne Cartier et épouse de Jean-Louis Dorais), Bella Kasch et Madeleine Boyer (amie de Thuribe Belzile) ${ }^{22}$. En pratique, JeuneLaurentienne est avant tout un groupe de rencontre et de discussion, mais ses membres participent également aux assemblées et autres activités publiques de Jeune-Canada. Il s'agit d'un groupe intrigant, mais dont les traces en archives sont malheureusement très limitées.

Le réseau est également caractérisé par la respectabilité de ses participants. Jeune-Canada bénéficie de la bienveillance de Lionel

${ }^{21}$ David Rajotte, Les jeunes laurentiens : jeunesse, militantisme et nationalisme dans le Canada français des années 1940, mémoire de maîtrise en histoire, Université de Montréal, 2006.

${ }^{22}$ Lettre de Madeleine Boyer à Lionel Groulx, 14 janvier 1936, BAnQ-M, Fonds Lionel Groulx, CLG1. Les liens de parenté ont été établis à la suite d'une recherche généalogique. 
Groulx, qui n'hésite pas à présenter le groupe comme les représentants de la jeunesse canadienne-française qui effectueront le relèvement de la nation ${ }^{23}$. Cette respectabilité favorise les contacts avec d'autres réseaux et la tenue d'activités extra-institutionnelles, des éléments importants pour la vitalité d'un réseau et pour son développement éventuel en mouvement. Jeune-Canada entretient de bonnes relations avec plusieurs autres groupes de jeunes, à commencer, comme le souligne l'historienne Karine Hébert, par les associations étudiantes de l'Université de Montréal ${ }^{24}$. Les membres Paul Dumas et Roger Larose sont respectivement présidents de l'Association des étudiants de la Faculté de médecine et de l'Association générale des étudiants de l'Université de Montréal. Le groupe a aussi ses entrées dans le journal étudiant Le Quartier latin, puisque Pierre Asselin en est l'administrateur, tandis que Paul Dumas, Roger Larose et Jean-Paul Verschelden y occupent des postes de direction. Le journal étudiant et la présence dans les associations étudiantes représentent de bons moyens pour susciter la participation des jeunes à leurs activités. Les relations entre Jeune-Canada et l'Association catholique de la jeunesse canadienne-française (ACJC) sont plus nébuleuses. Jeune-Canada reçoit d'abord l'appui de l'ACJC lors de sa première assemblée au Gesù, mais un conflit survient au cours de l'année 1933 entre Pierre Dansereau et Lionel Leroux, le président de l'ACJC. Ce dernier n'apprécie pas que Jeune-Canada organise une activité pour commémorer la fête de Dollard le même jour que son groupe ${ }^{25}$. Les tensions disparaissent le 23 février 1934 lorsque Jean-Paul Verschelden prend la présidence de l'ACJC. Ce dernier fait alors entrer d'autres membres de Jeune-Canada à la direction de l'ACJC, ce qui permet de conclure à une certaine forme de noyautage. Jeune-Canada bénéficie également

${ }^{23}$ Lionel Groulx, Mes mémoires, t. 3, Montréal, Fides, 1972, p. 276.

${ }^{24}$ Karine Hébert, La construction d'une identité étudiante montréalaise (1895-1960), thèse de doctorat en histoire, Université du Québec à Montréal, 2002, p. 200.

${ }^{25}$ Père Marquette, "Des patriotards qui se prennent aux cheveux! ", L'Autorité, 13 mai 1933, et Pierre Dansereau, "Au président général de l'ACJC ", Le Canada, 23 mai 1933. 
du soutien de la Société Saint-Jean-Baptiste de Montréal, qui lui prête le théâtre du Monument national pour certaines assemblées publiques, et de celui des Jésuites, pour la distribution de leurs publication $s^{26}$. Ces bonnes relations donnent au réseau une plus grande audience et une meilleure visibilité.

Lalliance la plus importante pour Jeune-Canada demeure celle que ses membres entretiennent avec le réseau groulxien. Plusieurs des amis de Lionel Groulx partagent sa bienveillance à l'endroit de Jeune-Canada. Omer Héroux, directeur du Devoir, écrit à quelques reprises un éditorial en première page pour féliciter et encourager le groupe $^{27}$. Pour sa part, le directeur de L'Action nationale, Arthur Laurendeau, accueille avec enthousiasme les articles des membres ${ }^{28}$. Jeune-Canada profite de l'aide et de l'appui des autres groupes nationalistes, car le groupe est considéré comme le réveil tant souhaité de la jeunesse canadienne-française. Sans nécessairement adhérer à l'ensemble des idées de Jeune-Canada, notamment celle de l'indépendance, le réseau groulxien encourage ses membres à les exprimer et à les diffuser. Il faut mentionner que les bases idéologiques de JeuneCanada sont semblables à celles de ses aînés. Le groupe souhaite valoriser la jeunesse, la nation canadienne-française, la langue française et le catholicisme. Les indépendantistes de 1932-1935 désirent améliorer le système en place en commençant par abolir les trusts, ce qui permettra, selon eux, de redonner la maîtrise de l'économie aux Canadiens français ${ }^{29}$. Lors d'une assemblée, Pierre Dansereau explique qu'il faut " nettoyer le capitalisme des trusts pour le sauver du socia-

${ }^{26}$ Guy Morin, "Cinq minutes avec Jean-Louis Dorais ", Le Quartier latin, 17 janvier 1935 , p. 4.

${ }^{27}$ Voir notamment Omer Héroux, "Bravo! Jeunes gens... ", Le Devoir, 19 décembre 1932, p. 1; Omer Héroux, "Continuez! ", Le Devoir, 20 décembre 1932, p. 1; Omer Héroux, "La plainte d'une génération ", Le Devoir, 24 avril 1933.

${ }^{28}$ Les membres de Jeune-Canada qui participent à L'Action nationale sont André Laurendeau, Dominique Beaudin, Jean-Paul Verschelden, Pierre Dansereau, Dollard Dansereau, Thuribe Belzile, René Monette, Paul Dumas et GeorgeÉtienne Cartier.

29 "Les Jeune-Canada dénoncent les trusts, en particulier ceux de l'électricité et de la gazoline ", Le Devoir, 14 novembre 1933, p. 4. 
lisme $e^{30} "$. Tout comme leurs aînés, les membres de Jeune-Canada estiment que le réveil de la nation canadienne-française se fera par un processus d'éducation nationale ${ }^{31}$. Sur le plan social, ils se méfient de l'arrivée massive de nouveaux immigrant ${ }^{32}$, ils considèrent que les femmes doivent, avant tout, être de bonnes mères de famille ${ }^{33}$, puis ils ne voient aucune contradiction entre l'action nationale et l'action catholique ${ }^{34}$. Ainsi, la grande différence entre les membres de Jeune-Canada et leurs aînés du Devoir et de L'Action nationale n'est pas idéologique; elle se situe plutôt dans le ton employé. En effet, les Jeune-Canada paraissent plus fougueux que les autres nationalistes groulxiens. Ils apportent peu de nuances à leurs propos et ils ne se gênent pas pour attaquer publiquement certains groupes et hommes politiques, que ce soient les juifs, les immigrants ou les libéraux de Taschereau. Les membres de Jeune-Canada sont ainsi des contestataires, mais de l'ordre établi, et non pas du milieu nationaliste groulxien pour lequel ils ont un grand respect. En ce sens, ils acceptent la censure des Jésuites et jamais ils n'oseraient se prononcer contre un Groulx ou un Minville ${ }^{35}$. Bref, les idées de Jeune-Canada sont plutôt fidèles à celles de ses aînés, mais elles sont peu nuancées, et les jeunes ne réussissent pas à transmettre leur désir d'indépendance à leurs prédécesseurs.

L'orientation vers l'indépendantisme se fait graduellement chez Jeune-Canada. Lors de la première assemblée au Gesù, en décembre 1932, le groupe croit toujours que la Confédération est potentiellement bonne. L'idée d'indépendance est introduite dans le réseau par André Laurendeau pendant l'été 1933, alors que le groupe est réuni pour

\footnotetext{
${ }^{30}$ Ibid.

${ }^{31}$ Jeune-Canada, Qui sauvera Québec?, Montréal, Le Devoir, 1935.

32 Dominique Beaudin, "À propos d'immigration ", L'Action nationale, octobre 1934, p. 148; Pierre Dagenais, dans Jeune-Canada, Politiciens et Juifs, Montréal, Le Devoir, 1933, p. 27.

${ }^{33}$ Bernard Hogue, dans Jeune-Canada, Qui sauvera Québec?, p. 81-82.

${ }^{34}$ André Laurendeau, Notre nationalisme, Montréal, Le Devoir, 1935.

${ }^{35}$ Morin, "Cinq minutes avec Jean-Louis Dorais ", p. 4.
} 
élaborer sa doctrine. Dans une lettre écrite à Lionel Groulx, André Laurendeau présente ses ambitions indépendantistes :

J'ai lu avec attention une enquête de L'Action française: "Notre avenir politique ». Cela m'a ouvert toutes sortes de perspectives. Cette idée m'était déjà chère. Je me demande si sa réalisation ne pourrait pas être notre œuvre. Au moment où vous étudiez cette question, vous vous contentiez de regarder venir les évènements. C'était juste, alors votre position était inattaquable. Ne vaudrait-il pas mieux, maintenant, aider les évènements ${ }^{36}$ ?

La doctrine de Jeune-Canada, écrite par Dominique Beaudin, Thuribe Belzile, Robert Charbonneau, Jean-Louis Dorais, André Laurendeau, Gilbert Manseau et Paul Simard, constitue un manifeste pour l'indépendance de la Laurentie ${ }^{37}$. Selon les auteurs, les nombreuses. disparités culturelles et les mentalités divergentes entre les deux peuples fondateurs de la Confédération empêchent le plein épanouissement des Canadiens français et représentent une menace à leur culture. Contrairement à Lionel Groulx, ils ne croient pas qu'une réforme de la Confédération puisse régler leurs problèmes. Ainsi préconisent-ils la formation d'un État francophone indépendant, situé sur les rives du Saint-Laurent, qui prendrait le nom de Laurentie.

À partir de l'été 1933, le réseau a défini son projet et la campagne est entamée. Pour diffuser leurs idées, les membres de Jeune-Canada ont recours à un répertoire d'actions. Ils écrivent des articles dans $L e$ Quartier latin, Le Devoir et L'Action nationale, organisent des assemblées publiques et des pèlerinages nationalistes, lancent une pétition nationale et animent des émissions de radio sur les ondes de CHLP et CKAC. En ce qui concerne plus précisément l'idée d'indépendance, celle-ci est progressivement proposée dans leurs tracts. L'objectif de cette série de six tracts est de livrer un message plus positif sur la question nationale et de donner une fierté nationale à la jeunesse.

${ }^{36}$ Lettre d'André Laurendeau à Lionel Groulx, 16 août 1933, BAnQ-M, Fonds Lionel Groulx, CLG1/A2143.

${ }^{37}$ "Projet de doctrine des Jeune-Canada », BAnQ-M, Fonds Jeune-Canada, CLG21. 
Dans le cadre de ses activités, le réseau montre une forte capacité de mobilisation. Si l'on se fie aux comptes rendus des événements dans la presse, les publications de Jeune-Canada ont en moyenne un tirage de 3000 exemplaires, tandis que leurs assemblées publiques attirent normalement plus de 1000 personnes, et parfois jusqu'à 3500 . De plus, sa pétition pour les droits de la langue française a récolté plus de 75000 signatures $^{38}$.

Bref, sous la période jeune-canadienne, le réseau indépendantiste est vigoureux et respecte plusieurs des préalables au mouvement social. En effet, le réseau a élaboré sa campagne, possède un répertoire d'actions diversifiées, détient une bonne unité interne, réalise des activités extra-institutionnelles, bénéficie d'une grande audience et d'une bonne capacité de mobilisation, puis ses leaders possèdent une bonne respectabilité. De plus, la conjoncture économique difficile entre 1932 et 1935 favorise l'émergence de nouvelles idées pour relever la nation. Malgré tout, le réseau indépendantiste ne se développe pas en mouvement. La principale raison est l'entêtement des membres du réseau à demeurer un petit groupe fermé d'une vingtaine de membres. Régulièrement, le secrétariat de Jeune-Canada reçoit des demandes d'adhésion, mais rares sont celles qui obtiennent une réponse positive. Le seul moyen d'être accepté est le réseautage, l'établissement d'un contact avec une personne déjà membre pour faire valoir sa candidature, et malgré cela, plusieurs demandes sont refusées. Dans son article, Denis Chouinard donne l'exemple de Robert Charbonneau qui, à deux reprises, tente de faire accepter dans les rangs de Jeune-Canada Claude Hurtubise, son collaborateur à $L a$ Relève, mais les deux fois on refuse de l'admettre dans le groupe, sans donner de raison pour justifier ce refus ${ }^{39}$. D'autre part, un groupe de jeunes nationalistes de Québec demande, en janvier 1934, la permission de mettre sur pied un comité de Jeune-Canada dans leur ville,

${ }^{38}$ Celle-ci n’a jamais été déposée, les signatures sont ainsi archivées dans le Fonds Jeune-Canada à BAnQ-M.

${ }^{39}$ Chouinard, " Des contestataires pragmatiques : les Jeune-Canada, 1932-1938", p. 22. 
mais cette demande est refusée ${ }^{40}$. Les membres de Jeune-Canada désirent demeurer le petit groupe d'amis qu'ils forment depuis leur passage au collège Sainte-Marie. Bien sûr, cela facilite la coordination et l'unité interne du réseau, mais empêche d'augmenter les effectifs, de renouveler les militants et de se développer hors de Montréal. Les membres de Jeune-Canada se perçoivent comme une élite qui étudie la question nationale et guide la jeunesse, mais ils n'envisagent pas, du moins pour l'instant, de prendre la direction d'une masse de jeunes indépendantistes. Bref, sous la période jeune-canadienne, le réseau ne désire tout simplement pas se développer en mouvement. C'est du moins l'opinion qu'avait André Laurendeau à l'été 1933, lorsqu'il présente à l'abbé Groulx ses ambitions indépendantistes : « Nous ne sommes pas encore prêts à lancer un tel mouvement, nous n'avons ni l'expérience ni les compétences qu'exigerait une pareille action. Il nous suffirait, à l'heure actuelle, d'étudier les problèmes nationaux à ce point de vue précis, et d'aller de l'avant, sans divulguer tout de suite nos ambitions ${ }^{41}$. » En 1935, André Laurendeau quitte pour l'Europe et les groupes de travail ne se réunissent plus, ce qui fait en sorte que Jeune-Canada met fin à ses activités. Les jeunes étudiants de 1932 font leur entrée sur le marché du travail et semblent davantage intéressés par leur carrière que par leur militantisme dans le réseau ${ }^{42}$. Et comme ils refusaient les nouvelles adhésions, aucune relève ne peut remplacer les militants désabusés. À son retour d'Europe en 1937, André Laurendeau tente de relancer Jeune-Canada avec de nouveaux membres, mais cette tentative se solde par un échec. Bref, 1935 marque un recul de l'influence de Jeune-Canada dans le réseau

${ }^{40}$ La demande d'accréditation à Jeune-Canada vient de Gérald Coote, Marcel Morin, Michel Dussault et Robert Darveau ("Les Jeune-Canada nient avoir une filiale à Québec ", Le Canada, 10 janvier 1934).

${ }^{41}$ Lettre d'André Laurendeau à Lionel Groulx, 16 août 1933, BAnQ-M, Fonds Lionel Groulx, CLG1/A2143.

${ }^{42} \grave{A}$ titre d'exemples, mentionnons que Dollard Dansereau est sous-ministre adjoint au Secrétariat de la province en 1936, Dominique Beaudin est nommé directeur de la Terre de chez nous en 1937, et que Gérard Filion est étu, la même année, secrétaire de l'Union des cultivateurs catholiques. 
indépendantiste, ce qui permet à d'autres organisations, beaucoup plus de droite, d'en prendre la tête.

\section{5-1939 : le réseau sous l'influence fasciste}

En 1934, un nouveau groupe s'intéresse à l'indépendance et gravite autour du réseau. Il est composé de jeunes de la région de Québec qui collaborent à la revue Vivre, dirigée par Pierre Chaloult, JeanLouis Gagnon et Philippe Vaillancourt. Vivre est une publication littéraire et politique qui paraît mensuellement de mai 1934 à mars 1935, puis hebdomadairement jusqu'en mai 1935. Lidéologie du groupe se base sur le corporatisme et l'anticommunisme. En ce qui concerne les autres idées, dont celle de l'indépendance, les avis sont partagés. Ainsi, quelques-uns des collaborateurs s'affichent-ils comme indépendantistes - Pierre Chaloult, Gérard Dagenais, Jean-Louis Gagnon - tandis que d'autres, particulièrement Jean-Charles Harvey, sont opposés à cette idée. Peu après la fondation du groupe, les administrateurs de Vivre éprouvent des problèmes pécuniaires et judiciaires. Ils choisissent de mettre fin à la parution de la revue et se réorientent vers l'édition de tracts favorables au fascisme, Les Cahiers noirs, mais un seul numéro est publié.

En février 1936, Pierre Chaloult, Jean-Louis Gagnon et d'anciens collaborateurs de Vivre, tels que Fernand Lacroix, Gabriel Langlais et Hélène Jobidon, se joignent à l'équipe de La Nation, un nouvel hebdomadaire de combat indépendantiste. Ses fondateurs sont de jeunes avocats de Québec : Paul Bouchard, Pierre Letarte, Albert Pelletier et Roger Vézina ${ }^{43}$. Pendant sa période indépendantiste, soit de février 1936 à mars 1937, La Nation paraît 58 fois. Bien que le journal soit publié jusqu'en 1939, le groupe de La Nation se détourne de son objectif initial au cours de l'hiver 1937 en empruntant la voie de l'autonomisme. La principale action de ce groupe indépendantiste demeure la publication de son journal de combat. À quelques

\footnotetext{
${ }^{43}$ Roger Vézina est l'associé de Paul Bouchard dans le même bureau d'avocats, tandis que Pierre Letarte est l'avocat de Vivre lors des poursuites de l'Hebdo-Laval.
} 
occasions, les membres tentent d'organiser des assemblées publiques ou d'établir une milice, mais ils remportent des succès mitigés.

À peu près au même moment, un second groupe fait son entrée dans le réseau indépendantiste à Montréal. Les Jeunesses patriotes sont fondées vers la fin de l'année 1935 par les frères Walter-Patrice et Dostaler O'Leary, deux jeunes journalistes qui reviennent d'un long séjour en Belgique où ils ont terminé leurs études ${ }^{44}$. Parmi les principaux membres des Jeunesses patriotes, mentionnons Michel Chartrand, Réal Denis, Hector Grenon, Jean Leduc, Émile Latrémouille, René Sarrazin, Maurice Sarrazin, Ivanhoé Valiquette et Philippe Vaillancourt. On retrouve également quelques membres de Jeune-Canada, groupe qui est alors, comme nous l'avons mentionné, quasi inactif. Outre Dostaler O'Leary, les autres de Jeune-Canada qui participent aux activités des Jeunesses patriotes sont Paul Dumas et Bernard Hogue. Tout comme le groupe de Québec, les Jeunesses patriotes sont de tendance fasciste et envisagent l'indépendance comme la première étape à l'établissement d'un régime corporatiste et nationaliste sur les rives du Saint-Laurent. Ils fondent eux aussi un journal de combat, L'Indépendance, mais y mettent fin après un seul numéro (février 1936). Ainsi, à partir de 1935, le réseau indépendantiste est-il constitué de deux groupes distincts, l'un à Québec et l'autre à Montréal, mais qui se rejoignent dans leur conception fasciste de l'indépendance.

Si les indépendantistes de 1932-1935 souhaitaient améliorer le régime politique en place, ceux de 1936-1939 le rejettent entièrement. Les membres de La Nation et des Jeunesses patriotes dénoncent le parlementarisme et la démocratie, deux concepts qu'ils jugent désuets. Ils considèrent que le parlementarisme libéral est un échec et écartent la solution du communisme, notamment à cause de son caractère

${ }^{44}$ Walter-Patrice et Dostaler O'Leary passeront toute leur vie à militer pour l'indépendance du Québec au sein de différentes organisations (Jeunesses patriotes, Chevaliers de la Table ronde, Rassemblement pour l'indépendance nationale et, dans le cas de Walter, le Parti québécois). 
internationaliste ${ }^{45}$. Pour répondre au désordre causé par la crise économique, ils privilégient une troisième voie, soit le corporatisme fasciste. Leurs influences corporatistes viennent surtout de Mussolini ${ }^{46}$, mais aussi d'intellectuels d'extrême droite tel que Charles Maurras ${ }^{47}$. Les indépendantistes entendent réorganiser la société du haut (chef unique et puissant) vers le bas (corporations de travailleurs). Ils n'hésitent pas à promouvoir la suppression de certaines libertés individuelles pour favoriser l'établissement d'un État fort et autoritaire qui pourra permettre le plein épanouissement de la nation canadiennefrançaise. Sur l'identité du chef, ils n'avancent pas de nom. Ils attendent l'avènement d'un véritable chef charismatique et autoritaire à l'image de Mussolini. Les autres idées dominantes dans le réseau indépendantiste sont alors l'opposition aux partis politiques ${ }^{48}$ et au droit de vote ${ }^{49}$ et certains souhaitent également suspendre l'immigra$\operatorname{tion}^{50}$. Concernant la place du religieux, précisons que les Jeunesses patriotes se qualifient d' "intégralement catholiques ${ }^{51}$ ", tandis que les membres de La Nation accordent peu d'importance à l'action catholique.

Par rapport à Jeune-Canada, les indépendantistes des années 1935-1939 ont de plus grandes ambitions pour le réseau. Premièrement,

${ }^{45}$ Jean-Louis Gagnon, "La potence d'abord ", La Nation, 16 juillet 1936, p. 1.

${ }^{46}$ Paul Bouchard, "Notre faillite commerciale ", La Nation, 7 mars 1936, p. 1.

${ }^{47}$ Sur l'influence de Charles Maurras auprès de certains indépendantistes des années 1930, voir Olivier Dard, " De la rue de Rome au Canada français : influences ou transferts? ", Mens : revue d'histoire intellectuelle de l'Amérique française, vol. VIII, n 1 (automne 2007), p. 7-66.

${ }^{48}$ Dostaler O'Leary, «Le canadianisme... voilà l'ennemi! », La Nation, 13 août 1936, p. 4. Notons néanmoins que les indépendantistes de 1936-1939 participent à quelques occasions au parlementarisme. Ils envoient des lettres à des députés pour encourager certains projets de loi, ils tentent de former un parti politique et de présenter des candidats et, lors des élections de 1936, ils appuient l'Union nationale.

49 Dans l'article suivant, Jean-Louis Gagnon explique que La Nation est opposée non seulement au droit de vote des femmes, mais aussi à celui des hommes: "Les femmes à barbe ", La Nation, 4 juin 1936, p. 4.

${ }^{50}$ Dans La Nation, Albert Pelletier est chargé de traiter de la question de l'immigration. Voir Albert Pelletier, "Xénophilie ", La Nation, 29 février 1936, p. 1.

${ }^{51}$ Les Jeunesses patriotes, "Assez de politicaillerie... de l'action ", L'Indépendance, février 1936, p. 2. 
ils souhaitent grossir leurs rangs et augmenter le nombre d'indépendantistes québécois. À Montréal, le comité central des Jeunesses patriotes, sous la présidence de Walter O'Leary, travaille à l'ouverture de "sections régionales » dans le but de diffuser les idées du réseau. Elphège Simoneau préside la cellule de Drummondville, J. B. Jalbert celle de Chicoutimi, Louis-Joseph Gagnon celle de Mont-Joli, alors que Jules Biron, puis Odilon Fortier s'occupent de la cellule de TroisRivières ${ }^{52}$. Nous retrouvons également des sections à Rouyn, à Hull, à Saint-Georges de Beauce, à Saint-Hyacinthe et à Alma. Pour sa part, Philippe Vaillancourt préside la cellule de Québec, la seule section des Jeunesses patriotes hors de Montréal qui semble réellement active. Vaillancourt, cofondateur de Vivre, préfere militer dans les Jeunesses patriotes plutôt que joindre les rangs de La Nation. Il forme une équipe avec des militants tels que Georges-Omer Blouin et Henri-Paul Papillon, puis, quelques mois plus tard, Jean-Louis Gagnon et Pierre Chaloult. Le groupe de La Nation tente lui aussi d'élargir ses rangs par la création des "Faisceaux d'action séparatiste ${ }^{53}$ ", mais cette milice, sous la responsabilité de Paul Talbot, ne connaît pas beaucoup de succès. Cette volonté de rejoindre les masses et de recruter de nouveaux membres à l'échelle provinciale permet de répertorier un plus grand nombre d'indépendantistes que dans la période précédente et, par le fait même, de constituer un réseau plus hétérogène qu'à l'époque de Jeune-Canada.

La campagne menée par le réseau indépendantiste est bien définie. Ses membres souhaitent l'indépendance nationale, mais contrairement à leurs prédécesseurs, ils ajoutent à la campagne des visées fascistes. Le répertoire d'actions pour la réalisation de cette campagne est assez diversifié. Le réseau comprend un journal hebdomadaire (La Nation) qui possède un tirage de base de 3000 exemplaires $^{54}$, puis les

${ }^{52}$ Lettre de Walter O'Leary à Philippe Vaillancourt, 3 mai 1936, BAnQ-M, Fonds O'Leary, CLG40/C2, 14.

${ }^{53}$ Paul Bouchard, "Fondation des Faisceaux d'action séparatiste ", La Nation, $1^{\text {er }}$ octobre 1936, p. 1.

54 "Dossier Jean-Louis Gagnon ", Division des archives de l'Université Laval, Fonds Paul Bouchard, P443/B2/1, 4. 
indépendantistes organisent des marches, des pèlerinages nationalistes et des débats publics. Les membres des Jeunesses patriotes sont assez créatifs et imaginent différents projets pour attirer l'attention et recruter de nouveaux membres. Par exemple, ils participent à la réalisation et à la commercialisation d'un buste à l'effigie de Lionel Groulx ${ }^{55}$, puis ils commandent la production d'un film de propagande en faveur de l'idée d'indépendance ${ }^{56}$.

Pour orienter leurs actions, les indépendantistes se dotent, en juillet 1936, d'un Comité central autonomiste, appelé à jouer un rôle de fédération des organisations indépendantistes. Les groupes signataires sont les Jeunesses patriotes, La Nation, la Jeunesse libérale nationale, l'Union nationale ouvrière et Jeune-Canada. Paul Bouchard est nommé président du comité, et La Nation en devient l'organe officiel. Hector Grenon, des Jeunesses patriotes, est responsable de l'organisation à Montréal, tandis que Jean-Louis Gagnon, de La Nation, est responsable de l'organisation à Québec ${ }^{57}$. Paul Bouchard explique que le Comité central autonomiste " est un organisme permanent de liaison entre les mouvements de jeunesse nationalistes en vue d'un front commun d'action et de pensée. C'est la tête du mouvement séparatiste [auquel] les principaux groupements de jeunesse nationaliste ont droit [de] mandater un représentant ${ }^{58}$ ". Le terme " autonomisme » plutôt que " séparatisme » est employé pour

${ }^{55}$ Les Jeunesses patriotes commercialisent un buste à l'effigie de Lionel Groulx, sculpté par l'artiste Jean-Jacques Cuvelier de Trois-Rivières. Voir « Un buste de l'abbé Groulx ", La Nation, 17 décembre 1936, p. 1.

${ }^{56}$ En 1937, Walter O'Leary est en contact avec un jeune cinéaste de Joliette, Christian Roy, pour réaliser un film de propagande en faveur de l'idée indépendantiste. Ce film de fiction, d'abord intitulé Roger le patriote et ensuite Un rebelle de 37, devait commémorer les rébellions de 1837-1838. Le scénario est écrit par Christian Roy, mais le film n'a jamais été réalisé, puisque ce dernier demande un budget de 25000 \$, ce qui est largement au-dessus des moyens dont disposent les Jeunesses patriotes. Voir la lettre de Christian Roy à Walter O'Leary, 11 janvier 1937, BAnQ-M, Fonds O'Leary, CLG40/C2, 13.

57 Paul Bouchard, "Le Comité central autonomiste ", La Nation, 20 août 1936, p. 2. 58 "Manifeste du Comité central autonomiste", juillet 1936[?], BAnQ-M, Fonds O'Leary, CLG40/C2, 6. 
rejoindre l'ensemble des groupes nationalistes au Québec et ainsi augmenter la portée du comité et le nombre de groupes membres. Malgré ce nom rassembleur, la direction est noyautée par des indépendantistes si bien que l'orientation du comité quant à la question nationale est déjà déterminée. L'expérience du Comité central autonomiste est intéressante, puisqu'elle reflète une tentative d'union du réseau en vue d'orienter les actions des indépendantistes partout au Québec. Les indépendantistes sont conscients qu'en réunissant leurs forces, ils augmentent leurs chances de réussite. Une telle fédération des organisations indépendantistes pour déterminer les visées du réseau laisse présager un pas de plus vers l'établissement d'un "mouvement indépendantiste ", mais le Comité central autonomiste ne durera que quelques mois en raison des nombreux conflits internes.

Pendant les années 1935-1939, donc, le réseau indépendantiste a de grandes ambitions et ses membres souhaitent sa transformation en mouvement, mais cet objectif échoue pour différentes raisons. Premièrement, depuis le recul de Jeune-Canada dans le réseau, les indépendantistes ne sont plus en mesure de tisser de forts liens et des contacts extra-institutionnels qui permettraient d'augmenter la portée de leurs messages. Alors que les activités de Jeune-Canada étaient couvertes par la plupart des grands quotidiens de l'époque, celles des indépendantistes des années 1935-1939 passent souvent inaperçues. Seuls L'Ordre (1934-1936) et L'Action catholique annoncent régulièrement leurs activités. De plus, les pages de L'Action nationale et du Devoir sont difficilement accessibles aux indépendantistes de $\mathrm{La}$ Nation et des Jeunesses patriotes. Lionel Groulx entretient une correspondance avec certains d'entre eux - Paul Bouchard, Pierre Chaloult, Marcel Hamel, Walter O'Leary - dans laquelle il offre ses conseils et recommandations, et il accepte parfois de participer à leurs activités et d'écrire des lettres d'encouragement pour leurs journaux. Cependant, l'abbé semble réticent à mettre ses relations au profit de ces jeunes. Ce changement d'attitude chez Lionel Groulx envers la jeunesse indépendantiste des années 1930 semble lié au critère de respectabilité 
des participants. Les membres de Jeune-Canada étaient pour la plupart issus de la petite bourgeoisie canadienne-française. Lionel Groulx connaissait certains d'entre eux depuis leur enfance, particulièrement André Laurendeau, le fils de son bon ami Arthur Laurendeau. JeuneCanada était en quelque sorte prédestiné à se développer dans le réseau du nationalisme groulxien. Les indépendantistes de 1935-1939, pour leur part, sont impliqués dans des scandales et des poursuites judiciaires. Par exemple, Vivre est poursuivi pour atteinte à la vie privée en 1935 par les rédacteurs du journal étudiant de l'Université Laval, l'Hebdo-Laval. Puis, lors des élections municipales de 1936 à Montréal, Réal Denis, des Jeunesses patriotes, est suspecté d'avoir participé à une bagarre ayant causé la mort d'un dénommé Fravas Gagalas ${ }^{59}$. D'autre part, les idées fascistes de ces indépendantistes sont moins facilement conciliables avec la pensée de Lionel Groulx que pouvaient l'être celles de Jeune-Canada. Pour ces différentes raisons, Groulx semble hésitant à accompagner ces jeunes dans leurs projets. Néanmoins, il continue à correspondre avec eux et à les conseiller, probablement parce qu'il se plaisait à recevoir autant d'estime de ces jeunes admirateurs, mais aussi parce qu'il se considérait comme un guide et un pédagogue pour la jeunesse canadiennefrançaise, en qui il voyait l'avenir de la nation.

À défaut de bénéficier des appuis qu'avait précédemment JeuneCanada, les indépendantistes de 1935-1939 s'efforcent de nouer des alliances régionales. Ainsi, à Trois-Rivières, les Jeunesses patriotes tentent-elles un rapprochement avec le conseil municipal ${ }^{60}$, tandis qu'à Rimouski, les indépendantistes reçoivent le soutien du notaire Eudore Couture $^{61}$, alors directeur de l'hebdomadaire local Le Progrès du Golfe. D'autres journaux semblent parfois favorables aux idées du

\footnotetext{
${ }^{59}$ Paul Bouchard, "L'affaire Gagalas : les mystères d'un meurtre politique ", $L a$ Nation, 31 décembre 1936, p. 1.

${ }^{60}$ Lettre d'Odilon Fortier à Walter O'Leary, 23 mars 1936, BAnQ-M, Fonds O'Leary, CLG40/C2, 11.

${ }^{61}$ Eudore Couture, "Les Jeunesses patriotes et leur objectif ", Le Progrès du Golfe, 9 octobre 1936 , p. 1.
} 
réseau, notamment L'Unité de Montréal et Le Travailleur de Worcester au Massachusetts ${ }^{62}$.

D'autre part, sans adhérer à l'ensemble des idées des Jeunesses patriotes et de La Nation, des nationalistes s'intéressent à la question de l'indépendance et appuient le réseau. C'est le cas notamment du député de l'Union nationale René Chaloult qui, sans se prononcer publiquement en faveur de l'indépendance, soutient financièrement les activités de son cousin Pierre Chaloult ${ }^{63}$. L'écrivain Claude-Henri Grignon, pour sa part, collabore à Vivre et à La Nation, puis défend le réseau à quelques reprises dans Les Pamphlets de Valdombre. Finalement, François Hertel (pseudonyme de Rodolphe Dubé) publie en 1936 Leur inquiétude $e^{64}$, le premier livre des années 1930 à aborder l'idée d'indépendance. Hertel présente lui aussi l'indépendance comme la solution au relèvement social et économique des Canadiens français, mais son approche s'inspire du personnalisme et du thomis$\mathrm{me}^{65}$. Ses idées contrastent avec celles des indépendantistes fascistes de 1935-1939. Bref, dans la seconde moitié des années 1930, le réseau possède toujours certains appuis, mais ceux-ci sont beaucoup moins importants que ne l'étaient ceux de Jeune-Canada.

\section{Un réseau divisé}

La principale faiblesse du réseau entre 1935 et 1939 est l'absence d'unité interne. Il se divise en différentes cliques, ce qui paralyse l'action collective des indépendantistes. En plus d'empêcher l'émergence d'un mouvement, ces divisions expliquent en grande partie la déroute du réseau vers la fin de la décennie. Tout d'abord, la transition entre la période jeune-canadienne et la période fasciste ne

${ }^{62}$ L'Unité, 14 février 1936, cité dans Jean-Louis Gagnon, "Les positions de L'Unité", La Nation, 4 juin 1936; "Les hommes et les journaux ", La Nation, 2 juillet 1936, p. 2.

${ }^{63}$ René Chaloult achète de l'espace publicitaire dans Vivre et La Nation.

${ }^{64}$ François Hertel, Leur inquiétude, Montréal, Éditions Albert Lévesque, 1936.

${ }^{65}$ Marie Martin-Hubbard, "Incursion personnaliste chez les thomistes canadiensfrançais des années 1930 et 1940 : l'exemple de François Hertel ", Mens : revue d'histoire intellectuelle de l'Amérique française, vol. VI, n 1 (automne 2005), p. 271-285. 
se fait pas sans heurt. En 1935, les indépendantistes de Vivre sentent le besoin de rompre avec les idées et les méthodes de Jeune-Canada. Jean-Louis Gagnon considère Jeune-Canada comme un cénacle inutile ${ }^{66}$, tandis que Fernand Lacroix décrit son président, Jean-Louis Dorais, comme un personnage d'un laconisme désespérant et critique le groupe pour son respect de l'ordre ${ }^{67}$. Les Jeunesses patriotes ont une plus grande estime de leurs prédécesseurs. Dans l'unique numéro de L'Indépendance, Walter O'Leary affirme prendre pour modèle le militantisme des membres de Jeune-Canada, "les précurseurs et les promoteurs du réveil national ${ }^{68}$ ". Le président des Jeunesses patriotes manifeste du respect envers le groupe de son frère aîné. En 1936, Dostaler O'Leary s'affiche toujours comme un membre de JeuneCanada, mais en réalité son militantisme s'inscrit davantage dans la mouvance des Jeunesses patriotes, puisque Jeune-Canada n'est presque plus actif.

Outre la discorde entre Jeune-Canada et Vivre, les indépendantistes de tendance fasciste, malgré leurs idées similaires, entretiennent de nombreux conflits internes. Premièrement, Walter O'Leary, président des Jeunesses patriotes, et Paul Bouchard, directeur de La Nation, rivalisent pour la direction du réseau indépendantiste. Selon O'Leary, Paul Bouchard a utilisé différentes méthodes, pas toujours légales, pour mettre fin aux Jeunesses patriotes. Dans une entrevue accordée à Raoul Roy en 1988, Walter O'Leary affirme avoir été enlevé et menacé par les militants de Paul Bouchard :

Un soir, comme je sortais du local de notre mouvement sur la rue Amherst, je me fais empoigner par quatre individus qui me mettent de force un bandeau sur les yeux et me poussent dans une automobile. La voiture part et j'ai l'impression que l'on s'en va vers le nord de la ville. On me fait sortir de là et on me fait monter au premier étage d'une maison. Lorsqu'on m'enlève le bandeau qui m'entourait la tête, je vois quantité de types avec

\footnotetext{
${ }^{66}$ Jean-Louis Gagnon, "Les Jeune-Canada ", Vivre, 26 avril 1935, p. 3.

${ }^{67}$ Fernand Lacroix, "Le Jeune-Canada parle ", Vivre, 12 avril 1935, p. 1.

${ }^{68}$ Walter O’Leary, [Sans-titre], L'Indépendance, février 1936, p. 3.
} 
des chemises noires et un foulard vert avec lequel ils se cachaient la figure. L'un d'entre eux, assis à une grande table, m'annonce qu'il représente les Faisceaux d'action séparatistes [sic] de Montréal (FAS). On me lit alors une déclaration que l'on voulait me faire signer et qui disait : Je soussigné, Walter O'Leary, président des Jeunesses patriotes, accepte par les présentes de démissionner en faveur de Paul'Bouchard qui deviendra le nouveau président du mouvement, à la suite de quoi ledit mouvement sera dissout et ses membres seront incorporés dans les FAS. En me retournant, je sens qu'on me pousse un objet dans les reins, et je découvre que c'est un pistolet. L'individu qui joue ce jeu me dit : tu signes ou je tire! [...] Finalement, ils m'ont laissé partir. Je découvre alors à qui appartenait la maison et que notre mouvement avait été infiltré par des gens du FAS ${ }^{69}$.

Pourtant, l'analyse de la correspondance entre Paul Bouchard et Walter O'Leary au début de l'année 1936 laisse entrevoir des relations plutôt amicales entre les deux hommes. Souvent, ils s'offrent des services mutuels et ne semblent pas se détester, malgré quelques mésententes à propos d'articles destinés à La Nation que Walter $\mathrm{O}^{\prime}$ Leary $^{70}$ aurait remis en retard. Même dans les années 1940, lorsqu'ils sont en Amérique latine, ils poursuivent leur correspondance et proposent de se rencontrer ${ }^{71}$. Il faut demeurer prudent avec cette histoire de l'ancien président des Jeunesses patriotes, qui a peut-être exagéré les faits pour rendre l'entrevue plus intéressante.

Le principal conflit dans le réseau indépendantiste oppose Paul Bouchard au trio de Vivre (Jean-Louis Gagnon, Pierre Chaloult et Philippe Vaillancourt) pour la direction de La Nation. En novembre 1936, les vivristes auraient contacté des collaborateurs du journal pour les convaincre de remplacer Paul Bouchard. Mis au courant, ce

${ }^{69}$ Raoul Roy, "Walter O'Leary des Jeunesses patriotes ", La Revue indépendantiste, automne 1988 , p. 38.

${ }^{70}$ Lettre de Paul Bouchard à Dostaler O'Leary, 21 août 1936, BAnQ-M, Fonds O'Leary, CLG40/C2, 15.

${ }^{71}$ Lettre de Paul Bouchard à Walter O'Leary, 8 mars 1941, BAnQ-M, Fonds O'Leary, CLG40/A4. 
dernier renvoie Gagnon et Chaloult et nomme Marcel Hamel rédacteur en chef, en remplacement de Pierre Chaloult ${ }^{72}$. Hélène Jobidon et Henri-Paul Papillon quittent La Nation en signe de protestation. Le conflit s'amplifie, puisque quelques jours avant cette tentative pour s'emparer de la direction du journal, Jean-Louis Gagnon, Pierre Chaloult et Henri-Paul Papillon se voient offrir des postes dans les Jeunesses patriotes par Walter O'Leary, ce qui laisse croire à Paul Bouchard que ce sont peut-être les Jeunesses patriotes qui ont tenté de le déloger ${ }^{73}$. Dans les faits, Paul Bouchard avait raison de se méfier des frères O'Leary, puisque ces derniers faisaient bel et bien partie $\mathrm{du}$ " complot». Le 4 novembre 1936, Henri-Paul Papillon mentionne à Dostaler O'Leary : "J'ai appris avec plaisir que tu étais convaincu qu'il n'y a rien à faire avec nos moineaux de La Nation. [...] Nous devons sortir notre journal pour le $1^{\text {er }}$ décembre ${ }^{74}$. " Le même jour, Philippe Vaillancourt décrit à Walter O'Leary ce à quoi ressembleraient les Jeunesses patriotes s'ils mettaient la main sur La Nation. Le secrétaire général serait Walter O'Leary, Jean-Louis Gagnon serait responsable des presses et de la propagande, Vaillancourt lui-même serait l'organisateur en chef, Henri-Paul Papillon s'occuperait des Faisceaux de Québec et Ivanhoé Valiquette de ceux de Montréal, puis Dostaler O'Leary serait le nouveau rédacteur en chef du journal ${ }^{75}$. Du côté de Paul Bouchard, il y a aussi des tentatives, et ce, dès le 2 octobre 1936, pour prendre la direction des Jeunesses patriotes. Dans le fonds d'archives de Paul Bouchard, on trouve la retranscription d'une réunion entre ce dernier et quelques amis ${ }^{76}$, au cours de laquelle

${ }^{72}$ Paul Bouchard, "Purge et sang nouveau à La Nation ", La Nation, 31 décembre 1936, p. 1.

${ }^{73}$ Lettre de Paul Bouchard à Walter O'Leary, 16 décembre 1936, BAnQ-M, Fonds O'Leary, CLG40/C2, 15.

${ }^{74}$ Lettre de Henri-Paul Papillon à Dostaler O'Leary, 4 novembre 1936, Division des archives de l'Université Laval, Fonds Paul Bouchard, P443/B2/1,4.

${ }^{75}$ Lettre de Philippe Vaillancourt à Walter O'Leary, 4 novembre 1936, Division des archives de l'Université Laval, Fonds Paul Bouchard, P443/B2/1,4.

${ }^{76}$ Les membres de la réunion utilisent des noms de code tels que Badin, Bistro, Codex et Micro. 
ceux-ci disent vouloir remplacer Walter O'Leary par Réal Denis et incorporer les Jeunesses patriotes comme aile militante de La Nation ${ }^{77}$. Bref, tant d'un côté que de l'autre, une volonté de conquérir le groupe rival dès l'automne de 1936 cause une grande scission dans le réseau indépendantiste. À partir de ce conflit interne, les relations se détériorent entre La Nation et les Jeunesses patriotes, et le Comité central autonomiste est aboli.

Toujours à la même époque, d'autres conflits secouent le réseau. Simone Jobidon, l'amie de Pierre Chaloult, quitte ce dernier pour fréquenter Walter O'Leary, ce qui mène à une dispute entre les deux hommes ${ }^{78}$. De plus, Réal Denis et Ivanhoé Valiquette, qui font tous deux partie du comité central des Jeunesses patriotes, exigent la démission l'un de l'autre puisqu'ils ne s'entendent pas sur les orientations que le groupe doit prendre ${ }^{79}$. Jean-Louis Gagnon, pour sa part, refuse de travailler dans le même groupe qu'Émile Latrémouille ${ }^{80}$, tandis que Philippe Vaillancourt est en conflit avec J. O. Martin ${ }^{81}$. L'année 1936 avait bien débuté avec un nombre record de journaux, de groupes et de militants en faveur de l'option indépendantiste, mais tous ces conflits internes, preuves d'une certaine immaturité, ont causé de nombreuses divisions et écarté le réseau de son objectif de promotion de l'indépendance.

À partir de 1937, le réseau indépendantiste décline. En mars, La Nation annonce à ses lecteurs qu'elle souhaite se rapprocher de l'idée groulxienne en délaissant l'option indépendantiste pour l'auto-

\footnotetext{
77 "Dossier Jean-Louis Gagnon ", Division des archives de l'Université Laval, Fonds Paul Bouchard, P443/B2/1,4.

${ }^{78}$ Lettre de Pierre Chaloult à Walter O'Leary, non datée, BAnQ-M, Fonds O'Leary, CLG40/A2.

${ }^{79}$ Lettre de Réal Denis à Ivanhoé Valiquette, 10 juin 1936, BAnQ-M, Fonds O'Leary, CLG40/C2, 18.

${ }^{80}$ Lettre de Jean-Louis Gagnon à Walter O’Leary, décembre 1936, BAnQ-M, Fonds O'Leary, CLG40/C2, 15.

${ }^{81}$ Lettre de Philippe Vaillancourt à Walter O'Leary, 16 décembre 1936, BAnQ-M, Fonds O'Leary, CLG40/C2, 14.
} 
nomisme $e^{82}$. Puis, les nombreux conflits internes ont considérablement nui aux Jeunesses patriotes. En 1938 et 1939, il ne reste qu'une quinzaine de membres réunis dans la cellule de Montréal (dirigée par Walter O'Leary et René Sarrazin) et celle de Québec (dirigée par Philippe Vaillancourt) pour tenter de donner un second souffle à l'organisation ${ }^{83}$. La principale activité des Jeunesses patriotes est alors l'édition de livres sur la question nationale, des ouvrages de Dostaler O’Leary, d'Émile Latrémouille et de Carmel Brouillard ${ }^{84}$. D'autre part, mentionnons la publication de l'abbé Wilfrid Morin en $1938^{85}$. Celui-ci rédige une thèse de doctorat en droit international à l'Institut catholique de Paris. Il étudie les droits et les conditions pour la nation laurentienne de se constituer en État souverain. Il s'agit probablement de l'ouvrage le plus complet portant sur l'indépendantisme écrit dans les années 1930, mais Wilfrid Morin n'entretient aucune relation avec les membres du réseau, ce qui en fait un " nœud externe ${ }^{86}$. Le déclenchement de la Seconde Guerre mondiale et le départ de Walter O'Leary, qui va étudier la philosophie à l'Université de Mexico, mettent définitivement fin au militantisme des Jeunesses patriotes et, par le fait même, à l'existence du réseau indépendantiste des années 1930 .

\section{$\sin$}

L'idée indépendantiste est présente au Québec dans les années 1930. Les individus et les groupes qui défendent cette thèse le font

\footnotetext{
${ }^{82}$ Marcel Hamel, "La main tendue ", La Nation, 18 mars 1937, p. 2.

${ }^{83}$ Lettre de Philippe Vaillancourt à Walter O'Leary, 17 janvier 1939, BAnQ-M, Fonds O'Leary, CLG40/C2, 14.

${ }^{84}$ Dostaler O'Leary, Séparatisme, doctrine constructive, Montréal, Éditions des Jeunesses patriotes, 1937; Émile Latrémouille, Tradition et indépendance, Montréal, Éditions des Jeunesses patriotes, 1939; Carmel Brouillard, Le séparatisme ne doit pas mourir, Montréal, Éditions des Jeunesses patriotes, 1939.

${ }^{85}$ Wilfrid Morin, Nos droits à l'indépendance politique, Paris, Guillemot et de Lamothe, 1938.

${ }^{86}$ Pour plus d'information à propos de Wilfrid Morin, voir Xavier Gélinas, "Wilfrid Morin ", dans Comeau, Courtois et Monière, Histoire intellectuelle de l'indépendantisme québécois, t. 1 : 1834-1968, p. 132-144.
} 
dans le cadre d'une action collective. Nous pouvons affirmer que le cycle de contestation correspondant à cette action collective est celui $\mathrm{du}$ réseau social. Les indépendantistes entretiennent des liens entre eux et souhaitent transmettre leurs idées à la société canadiennefrançaise, mais ils ne réussissent pas à rejoindre les masses et ainsi transformer leur réseau en mouvement social. Sous l'influence de Jeune-Canada, de 1932 à 1935, les liens entre les nœuds du réseau sont très forts. De plus, les indépendantistes répondent à plusieurs des critères établis par le sociologue Charles Tilly pour transformer le réseau en mouvement. Cependant, Jeune-Canada ne désire tout simplement pas ce changement. Ses membres ont choisi de demeurer un petit groupe d'une vingtaine d'amis intimes, empêchant ainsi le réseau d'augmenter ses effectifs et de s'étendre à l'extérieur de la région de Montréal. Les indépendantistes de 1935 à 1939, pour leur part, souhaitent rejoindre les classes populaires et transformer le réseau en mouvement politique. Toutefois, ils abordent l'idée d'indépendance par l'entremise d'une conception fasciste, une position radicale qui rebute plusieurs intellectuels de l'époque et qui nuit à la diffusion de leurs idées. De plus, les nombreux conflits internes et la division des indépendantistes en différentes cliques paralysent le réseau. Les indépendantistes de 1935-1939 semblent davantage préoccupés à lutter entre eux qu'à promouvoir l'idée d'indépendance.

Finalement, la conjoncture fait en sorte que les jeunes indépendantistes sont moins intéressés par la question nationale vers la fin de la décennie. Ce désabusement des jeunes est l'une des principales raisons de la disparition du réseau indépendantiste. À l'origine, le motif était de trouver une solution à la crise économique. L'indépendance était perçue comme le moyen pour établir un nouveau système en remplacement du capitalisme. À la fin des années 1930, la plupart de ces jeunes ont maintenant un emploi bien rémunéré. Serait-ce que certains d'entre eux ne sont plus si pessimistes à l'égard du capitalisme qui, finalement, semble résister à la crise économique? Suivant ce raisonnement, nous constatons que le réseau indépendantiste suit la même courbe que la crise économique. Le réseau nait véritable- 
ment à la fin de 1933, période la plus intense de la crise. En 1938, il perd beaucoup d'adeptes et d'influence en même temps que s'estompe la crise. Finalement, lorsque la Seconde Guerre mondiale stimule l'économie et amène la fin de la crise, le réseau disparaît. Il faut attendre après la guerre pour que l'idée indépendantiste refasse surface au Québec, où elle sera discutée et portée par les Jeunes Laurentiens et les Chevaliers de la Table ronde. Dans les années 1950 et 1960, un nouveau réseau indépendantiste émergera qui, lui, réussira à mettre en branle un mouvement. 